\title{
A TERNARY FUNCTION FOR DISTRIBUTIVITY AND PERMUTABILITY OF AN EQUIVALENCE LATTICE
}

\author{
IVAN KOREC
}

\begin{abstract}
The main result of the paper is
TheOREM 1. Let $A$ be a countable set and $L$ be a complete sublattice of the equivalence lattice on $A$. The following are equivalent

(i) $L$ is a distributive lattice of permutable equivalence relations.

(ii) There is an algebra with congruence lattice $L$ among the fundamental operations of which is a ternary function $f$ with the property

$$
f(a, b, b)=f(a, b, a)=f(b, b, a)=a
$$

for all $a, b \in A$.
\end{abstract}

This theorem is a contribution to the concrete representation problem for congruence lattices. Other results related to this problem can be found in [2]. We always assume that a complete sublattice of a complete lattice $U$ has the same extremal elements as $U$. Suppose $\vartheta$ is an equivalence relation on the set $A$ and $g$ is a function from some subset $X$ of $A^{3}$ into $A$. We write $(a, b, c) \vartheta(d, e, f)$ for $a \boldsymbol{\vartheta} d, b \vartheta e, c \vartheta f ; g$ is compatible with $\vartheta$ provided $g(a, b, c) \vartheta g(d, e, f)$ whenever $(a, b, c) \in X,(d, e, f) \in X$ and $(a, b, c) \vartheta(d, e, f) ; g$ is compatible with a set $L$ of congruence relations if it is compatible with each member of $L$. Analogous definitions are used for unary functions. In the proof of Theorem 1 we use:

THEOREM 2. Let $L$ be a complete distributive lattice of permutable equivalence relations on the countable set $A$. There is a function $f$ from $A^{3}$ into $A$ which is compatible with $L$ and for which (1) holds for all $a, b \in A$.

An analogous theorem, for $A$ arbitrary and $L$ finite, was proved in [3] ( $L$ was considered as a lattice of congruences of some algebra; however this fact was not substantially used). At the Colloquium on Universal Algebra in Oberwolfach, July 1973, A. F. Pixley asked whether the finiteness of $L$ can be omitted in his theorem. Our theorems give a partial answer to his question.

Proof of Theorem 2. For the sake of convenience we suppose that $A$ is countably infinite. The finite case may be obtained by halting our construction of $f$ below at the appropriate place, but this case was fully established in [3] (cf. Lemma 3.1 where the assumption that $L$ is the

Received by the editors January 28, 1976 and, in revised form, October 22, 1976.

AMS (MOS) subject classifications (1970). Primary 08A25.

(c) American Mathematical Society 1978 
congruence lattice of some algebra is extraneous). We may also suppose $A^{2} \in L$. Let

$$
D=\left\{(a, b, c) \in A^{3}: a, b, c \text { are pairwise different }\right\},
$$

$F=A^{3}-D$. Now $D$ is countably infinite, so we impose on it the order type of the natural numbers: $t_{0}<t_{1}<t_{2} \ldots$. Define

$$
S(a, b, c)=\{(d, e, f):\{d, e, f\} \underset{f}{\subsetneq} a, b, c\}\}
$$

and let

$$
g(a, b, c)=\left\{\begin{array}{ll}
a & \text { if } b=c \\
c & \text { otherwise }
\end{array} \text { for }(a, b, c) \in F\right.
$$

It is easy to verify that $g$ is compatible with all equivalence relations on $A$.

LEMMA 1. If the domain of $h_{k}$ consists of all $t<t_{k}$ with $t \in D$ and $h_{k} \cup g$ is compatible with $L$, then $h_{k}$ can be extended to $h_{k+1}$ with the domain $\left\{t_{i}\right.$ : $0 \leqslant i \leqslant k\}$ so that $h_{k+1} \cup g$ is compatible with $L$.

Proof of Lemma 1. Let $f_{k}=h_{k} \cup g$ and $E_{k}=\left\{t_{i}: 0 \leqslant i<k\right\} \cup S\left(t_{k}\right) . E_{k}$ is finite and $E_{k}$ is a subset of the domain of $f_{k}$. For each $t \in E_{k}$ let $\vartheta_{t}$ be the least equivalence relation in $L$ with $t \vartheta_{t} t_{k}$. Evidently $t \vartheta_{t} \vartheta_{s} s$ for all $t, s \in E_{k}$. Since $\boldsymbol{\vartheta}_{t} \vartheta_{s}=\vartheta_{t} \vee \vartheta_{s}$, we have $\vartheta_{t} \vartheta_{s} \in L$, so by the compatibility of $f_{k}$ we obtain $f_{k}(t) \vartheta_{t} \vartheta_{s} f_{k}(s)$. Consequently, by the Chinese Remainder Theorem (see Pixley [3] or Grätzer [1, p. 211, Exercise 68]) we conclude that there is $d \in A$ such that $f_{k}(t) \vartheta_{t} d$ for all $t \in E_{k}$. Let $h_{k+1}\left(t_{k}\right)$ be such a $d$. To see that $f_{k+1}$ $\left(=h_{k+1} \cup g\right.$ ) is compatible with $L$ suppose that $t \in E_{k} \cup F$ and $t \vartheta t_{k}$ for some $\vartheta \in L$. If $t \in E_{k}$ we have $\vartheta_{t} \subseteq \vartheta$ and $t \vartheta_{t} t_{k}$. But then $f_{k+1}(t)=$ $f_{k}(t) \vartheta_{t} h_{k+1}\left(t_{k}\right)=f_{k+1}\left(t_{k}\right)$, and so $f_{k+1}(t) \vartheta f_{k+1}\left(t_{k}\right)$. If $t \in F$ we can suppose $t=(a, a, b)$; the other two cases are similar. Let $t_{k}=(x, y, z)$. Then $\boldsymbol{x} \boldsymbol{\vartheta} a \boldsymbol{\vartheta} y$ and $z \boldsymbol{\vartheta} b$. So $x \boldsymbol{\vartheta} y$ and we obtain $(x, x, z) \vartheta t_{k}$. But $(x, x, z) \in$ $S\left(t_{k}\right) \subseteq E_{k}$. Hence

$$
f_{k+1}\left(t_{k}\right) \vartheta f_{k}(x, x, z)=z \vartheta b=f_{k+1}(a, a, b) .
$$

This completes the proof of Lemma 1.

By well ordering $A$ we can define $f$ by the following recursion using Lemma 1 to do the crucial step.

$h_{0}$ is the empty function;

$d_{k}$ is the least $e \in A$ such that $h_{k} \cup\left\{\left(t_{k}, e\right)\right\} \cup g$ is compatible with $L$;

$h_{k+1}=h_{k} \cup\left\{\left(t_{k}, d_{k}\right)\right\}$.

Then let $f=g \cup \cup_{k=0}^{\infty} h_{k}$. Observe that (1) holds since $g \subseteq f$. Let $t, s \in A^{3}$ and suppose $t \vartheta s$ for some $\vartheta \in L$. There is a $k$ such that $t$ and $s$ are both in the domain of $g \cup h_{k}$. Since $g \cup h_{k}$ is compatible with $L$ we conclude

$$
f(t)=\left(g \cup h_{k}\right)(t) \vartheta\left(g \cup h_{k}\right)(s)=f(s) .
$$

In this way Theorem 2 is established.

Proof OF TheOrem 1. It suffices to prove that (i) implies (ii); the converse was proved in [3, p. 183]. If (i) holds, then by Theorem 2 there is a function $f$ 
from $A^{3}$ into $A$ which is compatible with $L$ and which satisfies (1) for all $x, z \in A$; it will be one of the fundamental operations of the algebra which we construct. The remainder fundamental operations of it will exclude the equivalence relations not belonging to $L$.

LEMMA 2. If $\eta \notin L$ is an equivalence relation on $A$ then there is a unary operation $g$ on $A$ which is compatible with $L$ and not compatible with $\eta$.

Proof of Lemma 2. Let $\boldsymbol{\vartheta}_{(x, y)}$ be the least element $\xi$ of the lattice $L$ such that $x \xi y$. Then obviously $\eta \leqslant \bigvee_{(a, b) \in \eta} \vartheta_{(a, b)}$. However, $\eta \notin L$ and thus the equality does not hold. Therefore there is $\left(a_{0}, a_{1}\right) \in \eta$ such that $\vartheta_{\left(a_{0}, a_{1}\right)} \nless \eta$; hence there are $c_{0}, c_{1}$ such that $\neg c_{0} \eta c_{1}, c_{0} \vartheta_{\left(a_{0}, a_{1}\right)} c_{1}$. Take these $a_{0}, a_{1}, c_{0}, c_{1}$ and arbitrary $\xi \in L$. Then $a_{0} \xi a_{1}$ implies $\xi \geqslant \vartheta_{\left(a_{0}, a_{1}\right)}$ and, hence, $c_{0} \xi c_{1}$. Thus the unary partial function $g_{2}=\left\{\left(a_{0}, c_{0}\right),\left(a_{1}, c_{1}\right)\right\}$ is compatible with $L$; it obviously is not compatible with $\eta$. (Up to this moment we have used neither countability of $A$ nor (i).)

Impose on $A-\left\{a_{0}, a_{1}\right\}$ the order type of natural numbers $a_{2}<a_{3}<a_{4}$ $<\ldots$ Suppose that $k \geqslant 2$ and that $g_{k}=\left\{\left(a_{0}, c_{0}\right),\left(a_{1}, c_{1}\right), \ldots\right.$, $\left.\left(a_{k-1}, c_{k-1}\right)\right\}$ is constructed, $g_{k}$ is compatible with $L$. Let $\vartheta_{i}(0 \leqslant i<k)$ be the least element of $L$ such that $a_{i} \vartheta_{i} a_{k}$. In the same way as in Lemma 1 we can find the least $j$ such that $c_{i} \vartheta_{i} a_{j}$ for all $i<k$, and show that $g_{k+1}=g_{k} \cup$ $\left\{\left(a_{k}, c_{k}\right)\right\}$, where $c_{k}=a_{j}$, is compatible with $L$. Then $g=\cup_{k=2}^{\infty} g_{k}$ is also compatible with $L$. Since $a_{0} \eta a_{1}, \neg g\left(a_{0}\right) \eta g\left(a_{1}\right)$, the function $g$ is not compatible with $\eta$. Lemma 2 is established.

For every equivalence relation $\vartheta$ on $A, \vartheta \notin L$, let $g_{\vartheta}$ be a unary function compatible with $L$ and not compatible with $\vartheta$. Let the set of fundamental operations of an algebra $\mathbb{Q}$ consist of $f$ and all $g_{\vartheta}$; their ordering is not important. Then $L$ is the congruence lattice of $Q$. Q.E.D.

ReMARKs. 1. After $g_{2}$ was constructed in the proof of Lemma 2 we have not used $\eta$ in the construction of $g$. Hence we may ask that the algebra in (ii) has countable signature.

2. Using the first part of the proof of Lemma 2 we can show: If $A$ is an infinite set, $B$ is the set of all complete sublattices of the equivalence lattice on $A$, then $\operatorname{card}(B)=2^{\operatorname{card}(A)}$.

Acknowledgements. I am grateful to H. Draškovičová and M. Kolibiar for informing me of the problem and for their advice.

\section{REFERENCES}

1. G. Grätzer, Universal algebra, Van Nostrand, Princeton, N. J., 1968.

2. B. Jonsson, Topics in universal algebra, Lecture Notes in Math., vol. 250, Springer-Verlag, Berlin and New York, 1970.

3. A. F. Pixley, Completeness in arithmetical algebras, Algebra Universalis 2 (1972), 179-196.

Department of Algebra, Faculty of Sciences, University of Komenský, 81631 Bratislava, Czechoslovakia 